\title{
A importância da bioética na prática odontológica: considerações atuais da literatura
}

\section{The importance of bioethics in the dental practice: current considerations from the literature}

\author{
Jefferson David Melo de Matos* \\ André Guimarães Rodrigues** \\ Alessandra Dossi Pinto ${ }^{* * *}$ \\ Guilherme da Rocha Scalzer Lopes**** \\ Valdir Cabral Andrade
}

\section{Resumo}

Objetivo: revisar a literatura sobre os meios pelos quais o cirurgião-dentista deve orientar sua conduta, tornando-se ciente daquilo que lhe é exigido em relação à sua profissão. Materiais e método: esta revisão de literatura foi conduzida pelos principais bancos de dados de saúde: PubMed, SciELO e Google Scholar. As palavras-chave para a busca textual foram: ética profissional (ethics, professional); educação em odontologia (education, dental); odontologia (dentistry). Os critérios de inclusão foram: literatura que aborde a temática em estudo, literatura dos últimos anos, idiomas inglês e português, estudos laboratoriais e clínicos e revisão sistemática. Os critérios de exclusão foram: revisão de literatura, carta ao editor, artigo de opinião, literatura duplicada em bases de dados e literatura que não abordasse as variáveis em estudo. Revisão de literatura: a bioética baseia-se em quatro princípios básicos que estabelecem uma metodologia para analisar os casos concretos e os problemas éticos que ocorrem na prática da assistência à saúde, sendo eles: não maleficência, beneficência, respeito à autonomia e justiça. Sendo esses princípios fundamentais para o desenvolvimento da bioética, com isso, abordando uma forma peculiar de definir e manejar os valores envolvidos nas relações dos profissionais de saúde e seus pacientes. Considerações finais: a bioética deve nortear os avanços dentro do respeito ao ser humano e à sua individualidade, sem que ocorram possíveis infrações. Todavia, são necessários maiores estudos para uma maior compreensão sobre o tema e as melhores medidas para cada caso em particular.

Palavras-chave: Educação em odontologia. Ética professional. Odontologia.

\section{Introdução}

A fim de estabelecer limites de procedimento do profissional da saúde em relação ao paciente e às formas de sua atuação, em especial na área odontológica ${ }^{1}$, o Código de Ética Odontológico (CEO) apresenta normas que situam o cirurgião-dentista em seu âmbito profissional, deixando-lhe claro aquilo que constitui infração ética e qual deve ser o seu comportamento diante de sua profissão, estando o profissional ciente de suas responsabilidades perante o paciente e/ou a instituição na qual presta serviços ${ }^{2}$.

Ao consultar um paciente, o cirurgião-dentista deve, primeiramente, submetê-lo ao exame clínico, que tem por objetivo a obtenção de dados fundamentais para o diagnóstico. Além disso, para obtenção de um exame clínico preciso, é necessário que o cirurgião-dentista tenha uma acuidade assídua dos sentidos, capacidade de observação, critério e discernimento para cada caso, bom senso e conhecimento básico sobre as principais doenças e seus respectivos sinais e sintomas ${ }^{3}$. Esse, por sua vez, divide-se em duas fases: anamnese ou fase subjetiva, em que o paciente relata sua percepção dos sintomas e sua visão dos sinais; e exame físico ou fase objetiva, em que o profissional descreve, de forma detalhada, os sinais patognomônicos apontados pelo paciente 4 .

É de suma importância que o profissional redija o prontuário odontológico ou a ficha clínica do paciente, em que deve constar a identificação (nome, endereço, estado civil, identidade, entre outras in-

Graduado em Odontologia, Universidade de Vila Velha, Vila Velha, Espírito Santo, Brasil.

* Graduanda em Odontologia, Faculdade São Francisco de Assis, Santa Teresa, Espírito Santo, Brasil.

Doutorando em Odontologia Restauradora, Departamento de Prótese Dentária, Universidade Estadual Paulista Júlio de Mesquita Filho, São José dos Campos, São Paulo, Brasil.

${ }_{* * * * *}$ Professor Adjunto, Departamento de Odontologia, Universidade Federal de Juiz de Fora, Governador Valadares, Minas Gerais, Brasil. 
formações), o histórico médico e odontológico (atuais e progressos), os dados colhidos no exame clínico, que irão nortear o diagnóstico e um plano de tratamento adequado, assim como a sequência detalhada dos procedimentos clínico-cirúrgicos que serão realizados $^{5}$. O paciente tem total direito de posse de seu prontuário, sendo o dentista, ou qualquer outro profissional da saúde, impossibilitado de divulgar os dados sem o seu consentimento prévio e sem quebra da bioética ${ }^{6}$.

Vale ressaltar que a bioética não pode estar atrelada nem à intolerância, nem à moral do "não", ela deve, pelo contrário, apontar para o "fazer", mais do que para o "proibir"; deve discutir o que fazer, com quem, a quem, com quais meios, com que objetivos, de forma que as relações entre ética e ciência biomédicas sejam marcadas pela liberdade do homem e pelo respeito a todos os seres humanos ${ }^{7}$.

Este trabalho tem como objetivo fazer uma revisão da literatura sobre os meios pelos quais o cirurgião-dentista deve orientar sua conduta, tornando-se ciente daquilo que lhe é exigido em relação à sua profissão.

\section{Materiais e método}

Esta revisão de literatura foi conduzida pelos principais bancos de dados de saúde: PubMed, SciELO e Google Scholar. As palavras-chave para a busca textual foram: ética profissional (ethics, professional); educação em odontologia (education, dental); odontologia (dentistry). Os critérios de inclusão foram: literatura que aborde as temáticas em estudo, literatura dos últimos anos, idiomas inglês e português, estudos laboratoriais e clínicos e revisão sistemática. Os critérios de exclusão foram: revisão de literatura, carta ao editor, artigo de opinião, literatura duplicada em bases de dados e literatura que não abordasse as variáveis em estudo.

\section{Revisão de literatura}

\section{Princípios da bioética}

A bioética originou-se dos Estados Unidos da América, por volta da década de 1970, tornando-se mundialmente conhecida por estar baseada em quatro princípios, com dimensão universal e reconhecida como bioética principialista. O contexto da bioética foi criado para "identificar os princípios éticos que deveriam nortear as experimentações envolvendo seres humanos". Na realidade, portanto, os princípios foram "identificados" não para a bioética, mas, sim, para a ética referente às pesquisas biomédicas envolvendo seres humanos ${ }^{8}$.

Os quatro princípios que norteiam a bioética são apoiados na ideia de respeito, preservação dos direitos e conservação da vida humana: autonomia, não maleficência, beneficência e justiça ${ }^{9}$.

A autonomia é a capacidade de uma pessoa para decidir fazer ou buscar aquilo que ela julga ser o melhor para si, já o respeito à autonomia significa ter consciência do direito do cidadão de possuir um projeto de vida próprio, de ter seus pontos de vista e opiniões, de fazer escolhas autônomas, de agir segundo seus valores e convicções ${ }^{10}$.

A não maleficência diz que o profissional de saúde tem o dever de não causar mal e/ou danos ao seu paciente, devendo existir um mínimo ético, no quesito de um dever profissional, que, caso não seja cumprido, coloca o profissional de saúde diante de uma situação de prática negligente da medicina ou das demais profissões da área biomédica ${ }^{11}$.

A beneficência quer dizer fazer o bem; de uma maneira prática, isso significa a obrigação moral de agir para o benefício do outro ${ }^{12}$. Esse princípio, quando é utilizado na área de cuidados com a saúde, significa fazer o que é melhor para o paciente, não só do ponto de vista técnico-assistencial, mas também do ponto de vista ético. Usando todos os conhecimentos e habilidades profissionais a serviço do paciente, considerando, na tomada de decisão, a minimização dos riscos e a maximização dos benefícios quanto aos procedimentos que serão realizados ${ }^{13}$.

Ajustiça está associada, preferencialmente, com as relações entre grupos sociais, preocupando-se com a equidade na distribuição de bens e recursos considerados comuns, numa tentativa de igualar as oportunidades de acesso a esses bens ${ }^{14}$. O princípio de justiça, do ponto de vista filosófico, é explicado com o uso de vários termos; todos eles interpretam a justiça como um modo justo, apropriado e equitativo de tratar as pessoas em razão de alguma coisa que é merecida ou devida a elas ${ }^{15}$.

\section{Código de Ética Odontológico}

Ética na odontologia está diretamente ligada às normas e às leis estabelecidas pelo Conselho Federal de Odontologia (CFO), que estão reunidas em um Código de Ética (CE), tendo como objetivo direcionar a prática odontológica, atentando para os direitos e os deveres, assim como as possibilidades de se cometer infrações éticas, pelas quais o cirurgião-dentista, o profissional auxiliar da odontologia, a entidade, a instituição ou a operadora de planos de saúde poderá responder a um processo ético, resultando em condenação ou absolvição ${ }^{16}$.

Uma das premissas estabelecidas pelo Código de Ética é o de resguardar as informações do paciente, como pode ser observado neste trecho:

Art. 14. Constitui infração ética: I - revelar, sem justa causa, fato sigiloso de que tenha conhecimento em razão do exercício de sua profissão; II - negligenciar na orientação de seus colaboradores quanto ao sigilo profissional; e, III - fazer referência a casos clínicos identificáveis, exibir paciente, sua imagem ou qualquer outro elemento que o identifique, em qualquer meio de comunicação ou sob qualquer pretexto, salvo se o cirurgião-dentista estiver no exercício da docência ou em publicações científicas, nos quais, a autorização do paciente ou seu responsável legal, lhe permite a exibição da imagem ou prontuários com finalidade didático-acadêmicas ${ }^{17}$. 


\section{Infrações éticas e responsabilidade civil}

A ética baseia-se em valores morais e sociais, defendendo os direitos de todos. Infrações éticas estão ambientadas tanto no que se refere ao profissional da saúde com seu semelhante, no julgamento dos serviços, na "mercantilização" da prática da saúde, quanto na relação profissional/paciente, na falta de atenção e cordialidade, na má informação, no não respeito aos direitos e à autonomia ${ }^{18}$. Esses são exemplos práticos que estão presentes no cotidiano, mas se podem observar todos os parâmetros de infrações éticas nos códigos vigentes para cada esfera, que regulamentam e resguardam os direitos e os deveres dos cidadãos ${ }^{19}$. As infrações podem ser respondidas em diversas ordens, como a Justiça Trabalhista, o Código de Defesa ao Consumidor, Justiça Civil, Justiça Criminal ou em órgãos de regulamentação da profissão ${ }^{20}$. As responsabilidades profissionais e civis significam assumir o ônus decorrente da violação dos princípios éticos do que é prudente na prestação da assistência à saúde, além disso, o conhecimento do que é justo e necessário, não só no sentido ético ou moral, mas também dentro do sistema de obrigações e deveres, diante do que é lícito, devido e assegurado eticamente ${ }^{21}$.

\section{Normas para a publicação de caso clínico}

Algumas normas devem ser seguidas para que um caso clínico possa ser publicado. De início, é necessária a permissão para reprodução do material fotográfico do paciente ou retirado de outro autor, aprovando a utilização das imagens em periódicos científicos ${ }^{22}$. Em seguida, a provação do Comitê de Ética em Pesquisa (CEP), quando referente a pesquisas com seres humanos ${ }^{23}$. Além disso, é obrigatória a apresentação do número do protocolo de aprovação do CEP da instituição em que a pesquisa foi realizada ${ }^{24}$. Também se necessita de uma carta assinada por todos os autores afirmando o ineditismo e suas possíveis relações ou conflitos de interesse no trabalho, assim como a responsabilidade pelo conteúdo enviado ${ }^{25}$. Todas as pessoas designadas como autores devem ter participado suficientemente no trabalho para assumir responsabilidade pública pelo seu conteúdo ${ }^{26}$.

$\mathrm{O}$ crédito de autoria deve ser baseado somente em: contribuições substanciais para concepção e delineamento, coleta de dados ou análise e interpretação dos dados, redação ou revisão crítica do artigo em relação ao conteúdo intelectualmente significante e aprovação final da versão a ser publicada ${ }^{27}$. Os editores podem solicitar aos autores que justifiquem quando o total de autores exceder oito; não sendo permitida a inclusão de um novo autor após o recebimento da primeira revisão feita ${ }^{28}$; caso seja necessária, a inclusão deve ser declarada na seção de agradecimentos ${ }^{29}$.

\section{Procedimento odontológico}

Em razão da movimentada rotina clínica a que são submetidos os cirurgiões-dentistas, é indispensável a manutenção de toda a documentação referente ao atendimento executado nos pacientes, que se reveste de aspectos éticos e legais, já que tais registros constituem prova documental diante de pacientes insatisfeitos, que não hesitam em recorrer aos Conselhos Regionais de Odontologia ou até mesmo à justiça, devido à intensa atuação da mídia, que tem dedicado grande espaço ao chamado "erro médico" ${ }^{30}$, após a promulgação do Código de Defesa do Consumidor, que se caracteriza pelo exacerbado protecionismo ao consumidor - neste caso, o paciente -, que supostamente se apresenta como frágil, por não possuir um grau de conhecimento sobre o serviço que lhe está sendo fornecido ${ }^{31}$.

Esses documentos formam um conjunto de declarações firmadas pelo profissional, no exercício da profissão, servindo como provas e garantindo resguardo quanto a eventuais processos, podendo ser utilizados com finalidade jurídica, compostos de anamnese, consentimento livre e esclarecido, evolução clínica do tratamento, radiografias e fotografias do paciente, assim como as cópias de prescrições e $\operatorname{atestados}^{32}$.

\section{Prontuário}

O prontuário odontológico engloba todos os documentos necessários para que o cirurgião-dentista desempenhe de forma correta sua função, devendo conter informações relativas à saúde bucal e geral do paciente, auxiliando no diagnóstico e em eventuais demandas judiciais ou em casos de identificação humana $^{33-35}$. É elaborado pelo cirurgião-dentista, devendo conter, obrigatoriamente, a identificação do paciente e do profissional responsável, a anamnese, o odontograma com as informações iniciais, os exames complementares (como radiografias, tomografias, fotografias, modelos de estudo), as opções de tratamento, as informações pertinentes ao tratamento escolhido e à forma de pagamento, odontograma final, cópias dos documentos fornecidos ou emitidos ao paciente (como prescrições, orientações, atestados, contratos, recibos, declarações e termo de consentimento livre e esclarecido) e assinados pelos envolvidos $^{36-38}$.

O Código de Ética da Odontologia refere-se ao prontuário:

CAPÍTULO VII - DOS DOCUMENTOS ODONTOLÓGICOS - Art. 17. São obrigatórias a elaboração e a manutenção de forma legível e atualizada de prontuário e a sua conservação em arquivo próprio seja de forma física ou digital. Art. 18. Constitui infração ética: I - negar, ao paciente ou periciado, acesso a seu prontuário, deixar de lhe fornecer cópia quando solicitada, bem como deixar de lhe dar explicações necessárias à sua compreensão, salvo quando ocasionem riscos ao próprio paciente ou a terceiros ${ }^{17}$. 
O prontuário resguarda e assegura tanto o cirurgião-dentista quanto o paciente, que estão sujeitos a processos, penalidades e multas ${ }^{43}$.

\section{Conclusão}

A bioética deve nortear os avanços científicos e tecnológicos respeitando a individualidade do ser humano e, assim, minimizando as possíveis infrações éticas e morais. Nesse sentido, uma melhor compreensão sobre o tema favorecerá uma abordagem menos negligente para cada caso em particular.

\section{Abstract}

Objective: to review the literature on the means by which dentists should guide their behavior, becoming aware of the requirements concerning their profession. Materials and method: this literature review was performed by the main health databases of PubMed, SciELO, and Google Scholar. The keywords for the text search were ethics, professional; education, dental; dentistry. The inclusion criteria were the literature addressing the subject studied, the recent literature, English and Portuguese languages, laboratory and clinical studies, and systematic reviews. The exclusion criteria were literature reviews, letters to the editor, opinion articles, the literature duplicated in databases, and the literature that did not address the variables studied. Literature review: bioethics is based on four basic principles that establish a methodology for analyzing the concrete cases and ethical problems occurring in the health care practice, as follows: non-maleficence, beneficence, respect for autonomy, and justice. These are fundamental principles for the development of bioethics, hence addressing a particular way of defining and managing the values involved in the relationships between health professionals and their patients. Final considerations: bioethics should guide the advances regarding human beings and their individuality, without the occurrence of potential infractions. However, further studies are required for a better understanding of the subject and for the best measures for each particular case.

Keywords: Dental education. Professional ethics. Dentistry.

\section{Referências}

1. Gilson CC. Ethical and legal aspects of illustrative clinical recording. Br J Hosp Med 1994; 52(5):225-9.

2. Frizelle F. Consent for case reports and medical images. $\mathrm{N} \mathrm{Z}$ Med J 2009; 122(1300):8-10.

3. Lal S. Consent in dentistry. Pac Health Dialog 2003; 10(1):102-5

4. Cahana A, Hurst SA. Voluntary informed consent in research and clinical care: an update. Pain Pract 2008; 8(6):446-51.

5. Naidoo S. Informed consent for photography in dental practice. SADJ 2009; 64(9):404-6.

6. Casella C, Capasso E, Terracciano L, Delbon P, Fedeli P, Salzano FA, et al. Ethical and Legal Issues in Gestational Surrogacy. Open Med (Wars) 2018; 13:119-21.
7. Conti A, Capasso E, Casella C, Fedeli P, Salzano FA, Policino $\mathrm{F}$, et al. Blood transfusion in children: the refusal of Jehovah's witness parents'. Open Med (Wars) 2018; 13:101-4.

8. Garrocho-Rangel A, Cerda-Cristerna B, Pozos-Guillen A. Bioethical issues in conducting pediatric dentistry clinical research. J Clin Pediatr Dent 2018; 42(2):85-90.

9. Catalanotto FA. Expected changes in regulation and licensure: influence on future education of dentists. J Dent Educ 2017; 81(9):eS11-eS20.

10. Holden A. 'Blowing the whistle': the ethical, professional and legal implications of raising concerns and self-regulation within dentistry. Aust Dent J 2018; 63(2):150-5.

11. Dos Santos Silva W, Silveira RJ, de Araujo Andrade MGB, Franco A, Silva RF. Is the late mandibular fracture from third molar extraction a risk towards malpractice? Case report with the analysis of ethical and legal aspects. J Oral Maxillofac Res 2017; 8(2):e5.

12. Bawany MH, Padela AI. Hymenoplasty and Muslim patients: Islamic ethico-legal perspectives. J Sex Med 2017; 14(8):1003-10.

13. Reid KI. Informed consent in dentistry. J Law Med Ethics 2017; 45(1):77-94.

14. Bright E, D’Cruz L, Milne E. Consent - an update. Br Dent J 2017; 222(9):655-7.

15. Graziele Rodrigues L, De Souza JB, De Torres EM, Ferreira Silva R. Screening the use of informed consent forms prior to procedures involving operative dentistry: ethical aspects. $J$ Dent Res Dent Clin Dent Prospects 2017; 11(1):66-70.

16. Khoury BS, Khoury JN. Dentistry and criminal law. Aust Dent J 2017; 62(3):295-300.

17. Conselho Federal de Odontologia. Código de Ética Odontológico. Resolução CFO - 118/2012. Conselho Federal de Odontologia. Diário oficial da União. Resolução 42/2003.

18. Malpani S, Arora J, Diwaker G, Kaleka PK, Parey A, Bontala P. Child abuse and neglect: do we know enough? A cross-sectional study of knowledge, attitude, and behavior of dentists regarding child abuse and neglect in Pune, India. J Contemp Dent Pract 2017; 18(2):162-9.

19. Bracken-Roche D, Bell E, Macdonald ME, Racine E. The concept of 'vulnerability' in research ethics: an in-depth analysis of policies and guidelines. Health Res Policy Syst 2017; 15(1):8.

20. Dyer C. Surgeon suspended for 12 months after decision to strike him off was quashed. BMJ 2017; 356:j588.

21. Holden A, Dracopoulos SA. Owning the tooth: exploring the ethical and legal issues relating to the use of extracted human teeth in dental education in Australia. Aust Dent $\mathrm{J}$ 2017; 62(2):146-51.

22. Doubleday AF, Goben A. Exploring faculty knowledge and perceptions of copyright at U.S. dental schools: a pilot study. J Dent Educ 2016; 80(11):1308-18.

23. Cook L. Ethics in dentistry ... does it matter? Todays FDA $2016 ; 28(4): 20-2$.

24. Chambers DW. Moral communities and moral leadership. J Am Coll Dent 2015; 82(4):60-75

25. Hansen H. Undertreatment, an ethical issue. J Calif Dent Assoc 2016; 44(7):467.

26. American Dental Hygiene Association. Code of Ethics for Dental Hygienists. J Am Coll Dent 2015; 82(4):32-4.

27. Kenney JP. Child abuse and neglect: as dental professionals, how can we help and what are our responsibilities? Todays FDA 2016; 28(1):78-80. 
28. Lee A, Mortenson L. Thomson Reuters Accelus. Healthcare Workforce. Issue Brief Health Policy Track Serv 2015; 28:1113.

29. Almeida CAP. O prontuário odontológico e seus aspectos éticos e legais. CRO-Notícia 1984; 3(1):1-75.

30. Ulfsdotter Eriksson Y, Berg K, Boman UW, Hakeberg M. Contract care in dentistry: sense-making of the concept and in practice when multiple institutional logics are at play. Sociol Health Illn 2017; 39(7):1035-49.

31. Santos CP, Nogueira TH, Marson FC, Silva CO, Lolli MCGS, Lolli LF. Ética odontológica contemporânea - uma análise das contribuições do novo código deontológico da profissão. Braz J Surg Clin Res 2014; 8(2):24-30.

32. Machado MA, Daruge E, Paranhos LR. Estratégias de comunicação e o código de ética odontológica. Rev Clín de Ortodontia Dental Press 2013; 12(2)72-6.

33. Costa SS, Silva AM. O novo código de ética odontológica e as alterações no cotidiano do cirurgião-dentista. Odonto 2014; 22(43-44):71-81.

34. Garbin CAS, Amaral MA, Garbin AJÍ, Saliba TA. Análise lexical do Código de Ética Odontológica. Rev de Odontol da Unesp 2018; 47(2):79-84.

35. Peres H, Rodrigues BS, Paranhos LR, Fernandes MM. Aspectos éticos e legais relacionados à atuação dos técnicos e auxiliares em saúde bucal. Salusvita 2013; 32(3):36.

36. Cruz RM, Cruz CPAC. Gerenciamento de riscos na prática ortodôntica: como se proteger de eventuais problemas legais. Revista Dental Press de Ortodon e Ortoped Facial 2008; 13(1):141-56

\section{Endereço para correspondência:}

Guilherme da Rocha Scalzer Lopes

Rua Bernardino Monteiro, 700, Dois Pinheiros

29650-000, Santa Teresa, ES, Brasil

Telefones: (27) 99958-1702 - (88) 99603-9595

E-mail: matosjefferson19@gmail.com

Recebido: 27/06/18. Aceito: 31/07/18. 